\title{
Control of pathogenic effector T-cell activities in situ by PD-L1 expression on respiratory inflammatory dendritic cells during respiratory syncytial virus infection
}

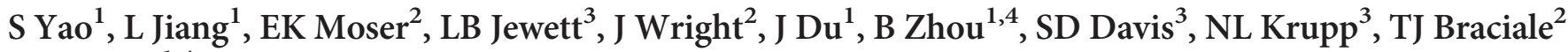 \\ and $J$ Sun $^{1,4}$
}

Respiratory syncytial virus (RSV) infection is a leading cause of severe lower respiratory tract illness in young infants, the elderly and immunocompromised individuals. We demonstrate here that the co-inhibitory molecule programmed cell death 1 (PD-1) is selectively upregulated on T cells within the respiratory tract during both murine and human RSV infection. Importantly, the interaction of PD-1 with its ligand PD-L1 is vital to restrict the pro-inflammatory activities of lung effector T cells in situ, thereby inhibiting the development of excessive pulmonary inflammation and injury during RSV infection. We further identify that PD-L1 expression on lung inflammatory dendritic cells is critical to suppress inflammatory T-cell activities, and an interferon-STAT1-IRF1 axis is responsible for increased PD-L1 expression on lung inflammatory dendritic cells. Our findings suggest a potentially critical role of PD-L1 and PD-1 interactions in the lung for controlling host inflammatory responses and disease progression in clinical RSV infection.

\section{INTRODUCTION}

Respiratory syncytial virus (RSV) is the most frequent cause of serious lower respiratory tract illness in infants and young children worldwide. ${ }^{1}$ In addition, RSV also leads to significant morbidity in elderly individuals and those with pre-existing conditions including chronic lung diseases and congenital heart diseases. ${ }^{2}$ Currently there is no approved RSV vaccine or efficacious treatment available for severe RSV-associated diseases, except a costly prophylactic antibody licensed only to high-risk infants. Emerging evidence from experimental models of RSV infection as well as clinical studies strongly suggest that the magnitude and features of the host inflammatory responses are important to lung injury and overall disease severity. ${ }^{3-7}$ Indeed, no firm correlation between disease severity and the levels of RSV replication was observed, ${ }^{8}$ suggesting that host inflammation may have a key role in determining host disease progression during RSV infection. As such, severe RSV-induced pulmonary disease is typically accompanied with an exaggerated inflammatory response in the lower respiratory tract, characterized by the overproduction of pro-inflammatory cytokines/chemokines and increased infiltration of inflammatory cells. ${ }^{4,7,9}$ Hence, control of pulmonary inflammation and the suppression of excess tissue injury might represent a novel approach to limit the pathology from RSV infection.

The exact mechanisms underlying the development of severe pulmonary inflammation and diseases following RSV infection are not fully understood. Since severe pulmonary inflammation is only observed in a small fraction of patients, it is estimated that variation (polymorphisms) among host factors regulating inflammation may be critical in determining the severity of inflammation and disease outcome., ${ }^{7,9}$ Notably, the host immune and inflammatory responses are tightly regulated by various immune-regulatory factors including specialized cell

${ }^{1}$ Department of Pediatrics, Herman B. Wells Center for Pediatric Research, Indiana University School of Medicine, Indianapolis, Indiana, USA. ${ }^{2}$ Beirne B. Carter Center for Immunology Research, University of Virginia, Charlottesville, Virginia, USA. ${ }^{3}$ Pediatric Pulmonology, Allergy and Sleep Medicine, Department of Pediatrics, Indiana University School of Medicine, Indianapolis, Indiana, USA and ${ }^{4}$ Department of Microbiology and Immunology, Indiana University School of Medicine, Indianapolis, Indiana, USA. Correspondence: J Sun (sun32@iupui.edu)

Received 25 April 2014; accepted 21 September 2014; published online 3 December 2014. doi:10.1038/mi.2014.106 
types (e.g. Foxp $3^{+}$regulatory T cells $($Treg)) and immuneregulatory cytokines (e.g. IL-10)). Studies in the murine model of RSV infection have revealed that Treg cells have a critical role in limiting severe inflammation and immunopathology during infection. ${ }^{10-13}$ Similarly, effector T-cell derived IL-10 has also been demonstrated to counter-balance host inflammatory responses and suppress severe pulmonary injury. ${ }^{14-16}$ Interestingly, allelic polymorphisms in the IL-10 locus are correlated with the risk of the development of severe bronchiolitis in RSVinfected children. ${ }^{17,18}$ Together these data suggest the possibility of preventing the development of excessive lung injury during RSV infection through the selective engagement and/or manipulation of immune-regulatory pathways.

Programmed cell death 1 (PD-1) protein is a T-cell coinhibitory receptor that belongs to the $\mathrm{CD} 28$ family. Unlike the interaction of $\mathrm{CD} 28$ and its ligands, $\mathrm{B} 7$ molecules, the interaction of PD-1 with its ligands, PD-L1 and PD-L2, delivers potent inhibitory signals to activated $\mathrm{T}$ cells. Hence, the interaction between PD-1 and its corresponding ligands is important for maintaining tissue homeostasis and preventing the development of autoimmune diseases. ${ }^{19}$ Conversely, the PD-1 pathway has also been shown to allow pathogen persistence during chronic viral infections by suppressing the efficient development of T-cell responses. ${ }^{20-22}$ The roles of PD-1 and its ligands in acute virus infection are less clear. ${ }^{23}$ Recently, it was demonstrated that the blockade of PD-1 and PD-L1 interaction in vivo resulted in elevated host effector T-cell responses and enhanced viral clearance during influenza and human metapneumovirus (HMPV) infection, suggesting that PD-1 and PD-L1 may also play a role in restricting effective anti-viral T-cell responses during acute viral infections. ${ }^{24-26}$ Similarly, in an in vitro co-culture model, the PD-L1/PD-1 interaction has been shown to inhibit $\mathrm{CD}^{+}{ }^{+} \mathrm{T}$ cell effector function when encountering RSV-infected human epithelial cells. ${ }^{27}$ However, the exact function of PD-1 and its ligands in vivo during RSV infection, particularly within the respiratory tract, is currently unknown.

We have investigated the role of PD-1 and PD-1 ligand interaction in regulating RSV pathogenesis and host immune responses in the lung during experimental RSV infection. We found that $\mathrm{T}$ cells from RSV-infected murine lungs, in particular IL-10-expressing effector T cells, expressed high levels of PD-1 compared with their counterparts in the secondary lymphoid organs. Interestingly, human $\mathrm{T}$ cells isolated from the respiratory tract of RSV-infected subjects also expressed higher levels of PD-1 than circulating T cells. In the murine model, blockade of the PD-1 and PD-L1 interaction in vivo, at the time of T-cell infiltration into the lung, resulted in enhanced pulmonary inflammation and lung injury but had only a modest effect on viral clearance. We found that PD-1/ PD-L1 blockade enhanced the pro-inflammatory activity of effector T cells independent of the action of IL-10 and Treg cells. We demonstrated that lung inflammatory dendritic cells (DCs) are the main cellular sources of PD-L1 responsible for the inhibition of effector T-cell activities. Furthermore, a signaling pathway involving type I and II interferons (IFNs)-STAT1-
IRF1 was critical for the upregulation of PD-L1 on inflammatory DCs. Our analysis has revealed a critical role of IFNdependent PD-L1 expression on lung inflammatory DCs in restricting excessive pulmonary inflammation and injury during RSV infection.

\section{RESULTS}

\section{PD-1 expression on respiratory $\mathrm{T}$ cells during murine and human RSV infection}

To define the role of PD-1 in regulating T-cell function in the lungs during RSV infection, we first examined the kinetics of PD-1 expression on lung $\mathrm{CD}^{+}{ }^{+}$and $\mathrm{CD} 8{ }^{+} \mathrm{T}$ cells following RSV infection of mice. We found that percentages of PD-1expressing cells among lung $\mathrm{CD} 4^{+}$and $\mathrm{CD} 8{ }^{+} \mathrm{T}$ cells, and PD-1 expression (mean fluorescence intensity) per cell were markedly increased at days 5 and 7 post RSV infection, the time when RSV-specific T cells begin infiltrating to the lungs (Figure 1a,b and Supplementary Figure S1A online). Compared with lung $\mathrm{T}$ cells, we detected only very modest PD-1 expression on activated T cells from draining mediastinal lymph nodes (MLN, Figure 1c), suggesting that PD-1 expression is primarily restricted to $\mathrm{T}$ cells at the site of infection. We recently demonstrated that effector $\mathrm{CD} 4{ }^{+}$and $\mathrm{CD}^{+}{ }^{+} \mathrm{T}$ cells in the infected lungs express the regulatory cytokine IL-10 during RSV infection. ${ }^{16}$ Interestingly, we found higher PD-1 expression on IL-10 producing lung effector $\mathrm{CD}^{+}$and $\mathrm{CD}^{+}{ }^{+} \mathrm{T}$ cells than on activated IL-10 nonproducing lung $\mathrm{CD}^{+}{ }^{+}$and $\mathrm{CD} 8{ }^{+} \mathrm{T}$ cells (Figure 1d).

In conjunction with this analysis, we examined whether PD1 is expressed on pulmonary T cells following human RSV infection. We compared the expression of PD- 1 on T cells from peripheral blood and nasal washes of children with RSV infection $(n=6)$ and from peripheral blood of uninfected healthy children $(n=4)$. We found that PD-1 expression was significantly upregulated on $\mathrm{CD} 8{ }^{+} \mathrm{T}$ cells from the nasal washes of RSV-infected patients compared with $\mathrm{CD} 8{ }^{+} \mathrm{T}$ cells from the subjects' peripheral blood (Figure 1e,f). We also examined PD-1 expression on $\mathrm{CD} 4{ }^{+} \mathrm{T}$ cells and observed a trend toward PD-1 upregulation on $\mathrm{CD} 4{ }^{+} \mathrm{T}$ cells in the nasal washes, which did not reach statistical significance possibly due to a limited sample size (Supplementary Figure S1B). Nevertheless, these data suggest that human airway T cells, in particular CD8 ${ }^{+} \mathrm{T}$ cells, express PD-1 during RSV infection.

\section{PD-L1 blockade exacerbated pulmonary inflammation and host diseases during RSV infection}

We next sought to determine the function of PD-1 on lung $\mathrm{T}$ cells during RSV infection. We blocked the interaction of PD-1 with its ligands PD-L1 or PD-L2 by injection of $\alpha$-PD-L1 (clone: 10B5) or $\alpha$-PD-L2 (clone: Ty25) mAb at the time of T-cell infiltration to the lungs (i.e. day 4 and 6 post infection). We chose these time points to specifically block PD-1 and PDL1 interaction in the lung, rather than to inhibit initial PD-1 and PD-L1 interaction during T-cell priming in the mediastinal lymph nodes at the early days following infection (i.e. days 1-4 post infection) since T-cell activation during priming can 


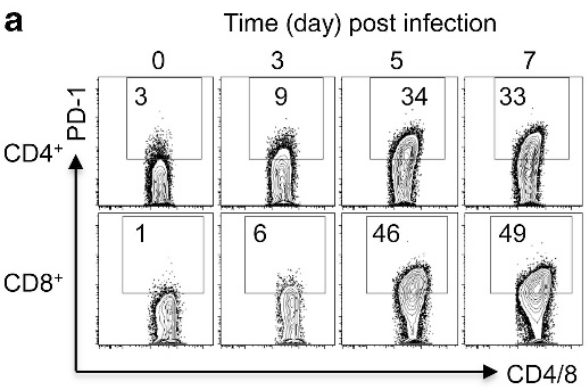

b

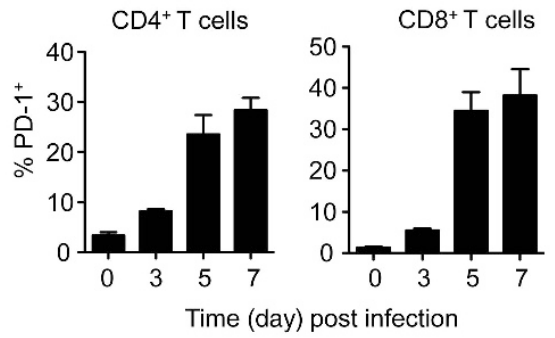

C

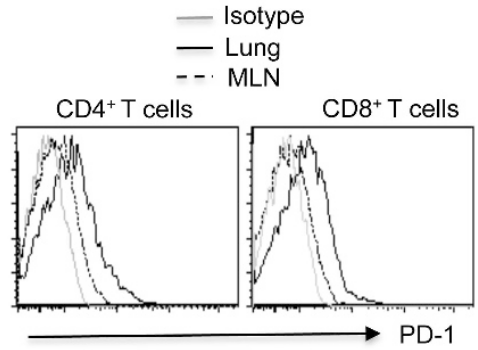

d $\quad \mathrm{CD}^{+} \mathrm{T}$ cells

CD8 ${ }^{+} \mathrm{T}$ cells
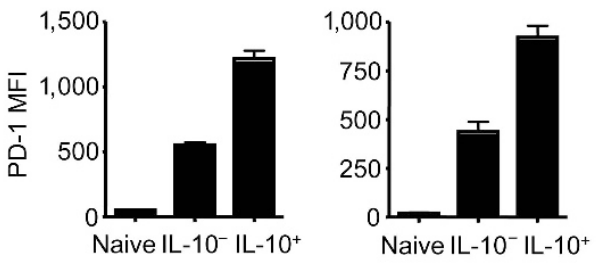

e
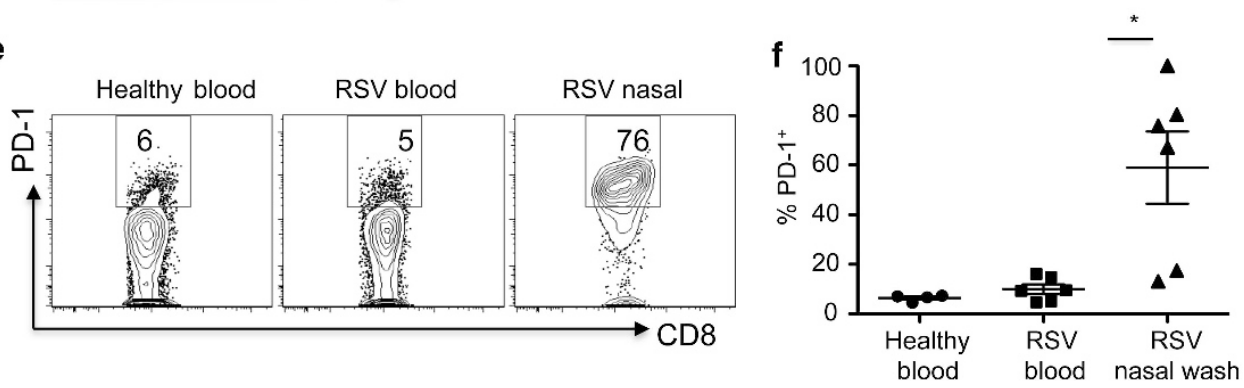

Figure 1 Murine and human T cells express programmed cell death 1 (PD-1) within the respiratory tract during respiratory syncytial virus (RSV) infection. (a-c) Wild-type Balb/c mice were infected with RSV. PD-1 expression (a) and \% of PD- $1^{+}$cells in CD4 ${ }^{+}$and CD8 ${ }^{+} \mathrm{T}_{\text {cells }}(\mathbf{b})$ at different days post infection are depicted. (c) PD-1 expression on activated CD4 ${ }^{+}$and CD8 ${ }^{+}$T cells (CD44 ${ }^{\text {hi }}$ ) of lung and mediastinal lymph nodes at day 5 post RSV infection. (d) IL-10-eGFP reporter (Vert-X) mice were infected with RSV. Mean fluorescence intensity (MFI) of PD-1 expression on naïve, IL-10 ${ }^{-}$, or IL$10^{+}\left(\right.$CD $\left.44^{\text {hi }}\right)$ lung T cells is depicted (day 5 post infection). (e, f) Peripheral blood mononuclear cells and nasal washes were collected on healthy children or RSV-infected children. (e) PD-1 expression on CD8 ${ }^{+}$T cells is depicted. (f) $\%$ of PD-1 ${ }^{+}$cells in CD8 ${ }^{+}$T cells is depicted. (a-d) Data are representative of two to three independent experiments. (e, f) Data are from four healthy and six RSV-infected donors. ${ }^{*}, P<0.05$ as determined by nonparametric Mann-Whitney test.

transiently upregulate PD-1. ${ }^{19,28}$ We found that injection of blocking PD-L1 Ab significantly enhanced host weight loss during RSV infection (Figure 2a), suggesting that the PD-L1/ PD-1 interaction is vital for restricting host morbidity. In contrast, we observed only moderate enhancement of host morbidity following PD-L2 blockade (Supplementary Figure S2A), presumably due to the lower levels of PD-L2 expression in the lung compared with the expression of PD-L1 (Supplementary Figure S2B). These data indicated that PD-L1/PD-1 interaction, but not PD-L2/PD-1 interaction, is critical to suppress the development of severe host disease during RSV infection. In parallel, we examined viral replication by determining RSV-L gene expression in the lung and RSV titers in the airway following either Rat immunoglobulin-G (IgG) or $\alpha$-PD-L1 treatment. We found that both viral genome content in the lungs (Figure $\mathbf{2 b}$ ) and virus titers in the airway (Figure 2c) of mice treated with $\alpha$-PD-L1 were comparable to those of control mice, suggesting that enhanced host morbidity following PD-L1 blockade is not due to enhanced viral replication in the lung.

We next sought to determine the extent of lung inflammation/injury following PD-L1 blockade during RSV infection. To this end, we examined vascular leakage into the lung airway by Evans-Blue assay. ${ }^{29}$ We found that mice exhibited significantly enhanced Evans-Blue extravasation to the airways following $\alpha$-PD-L1 treatment (Figure 2d), suggesting that these mice had enhanced vascular and airway permeability and injury. Enhanced non-neutralizing RSV-specific Ab production has been linked to lung damage in secondary RSV infection following immunization of formalin-inactivated virus, most probably due to the insufficient T-cell help. ${ }^{30,31}$ However, mice received $\alpha$-PD-L1 exhibited moderately enhanced follicular T-helper cell (Tfh, a specialized T-helper cell type responsible for helping $\mathrm{B}$ cells ${ }^{32}$ ) generation, comparable germinal center $\mathrm{B}$ cell formation and similar serum, and/or airway RSV-specific IgG levels to mice received control Ab during primary RSV 

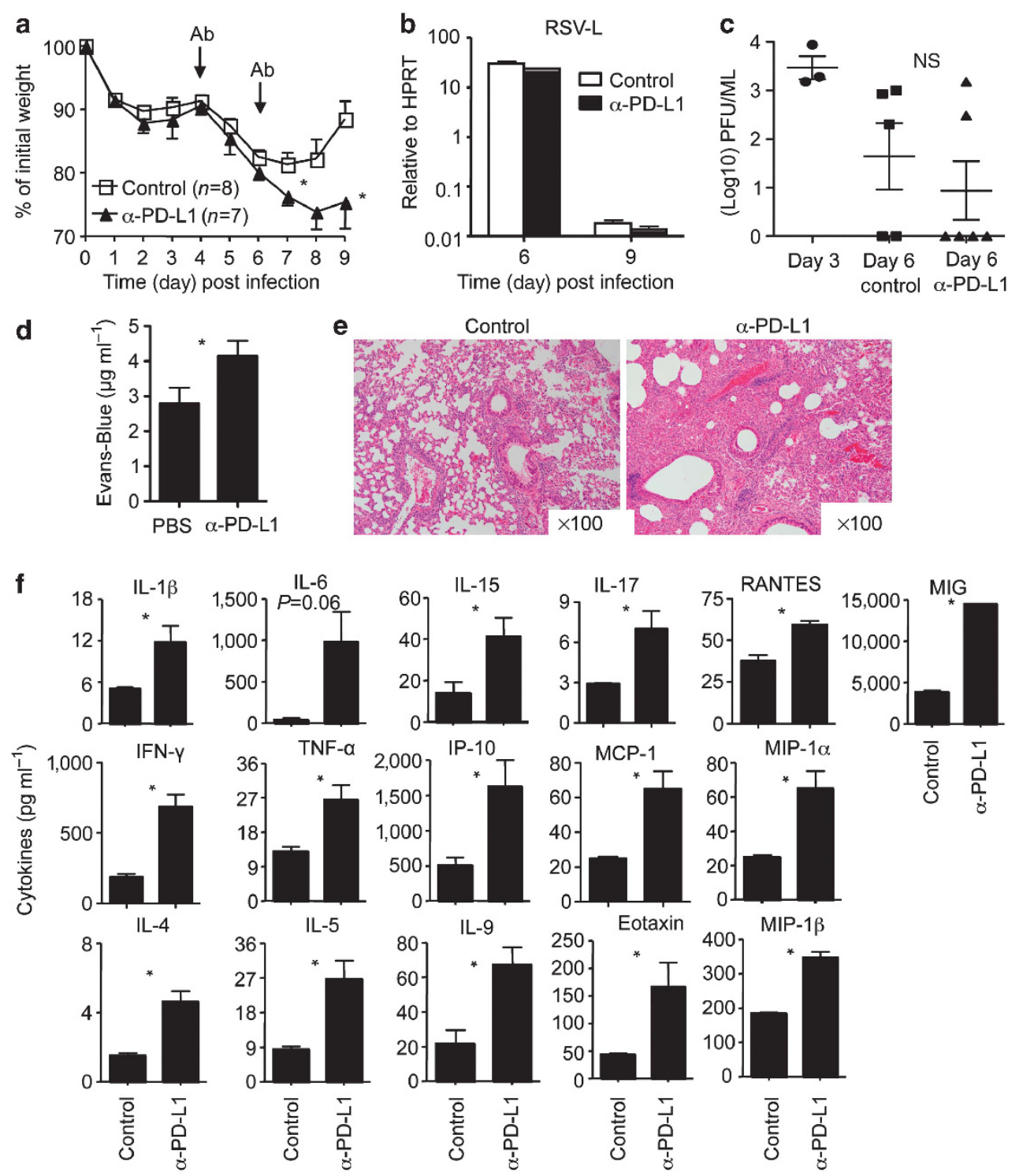

Figure 2 Programmed cell death 1 (PD-1) blockade following respiratory syncytial virus (RSV) infection leads to enhanced host morbidity and pulmonary injury. Wild-type Balb/c mice were infected with RSV and treated with control antibody, phosphate-buffered saline (PBS) or $\alpha$-PD-L1 as indicated. (a) Host morbidity was monitored through weight loss. (b) RSV replication in the lung was monitored through RSV-L gene expression at indicated days post infection. (c) Airway virus titers from indicated mice were determined by plaque assay. Day 3, airway RSV titers from day 3-infected mice; day 6 control, airway RSV titers from day 6-infected mice treated with Rat IgG; day $6 \alpha$-PD-L1, airway RSV titers from day 6 -infected mice treated with $\alpha$-PD-L1. (d) Lung vascular and airway leakage was monitored through Evans-Blue concentrations in the airway at day 7 post infection. (e) Lung histopathology was measured by hematoxylin and eosin staining at day 7 post infection. (f) Pro-inflammatory cytokine levels in BAL from control or $\alpha$-PDL1-treated mice (day 9 post infection). Data are pooled from two to three experiments $(\mathbf{a}, \mathbf{c})$, representative from two to three experiments, (b, $\mathbf{d}, \mathbf{e})$ or from one experiment with four to five mice each group (f). ${ }^{*}, P<0.05$ was determined by unpaired Student's $t$-test (a, d, f), NS, non-significant.

infection (Supplementary Figure S3), indicating that the development of enhanced lung damage is unlikely due to the enhanced deposition of RSV-specific IgG. We also examined lung injury by lung morphology in hematoxylin and eosin staining of lung sections from RSV-infected mice. We found that lungs from $\alpha$-PD-L1-treated mice demonstrated increased infiltration of inflammatory cells to the interstitium and augmented airway wall thickness (Figure 2e). In addition, we surveyed mice at day 9 post infection for the expression of a panel of inflammatory mediators in the broncho-alveolar lavage fluid (BALF) following treatment with $\alpha$-PD-L1 or control antibody. We found that a number of these mediators were elevated in the airway of $\alpha$-PD-L1-treated mice compared with those of mice that received control Ab (Figure 2f). Consistent with increased cytokine and chemokine levels in the respiratory tract, we observed increased numbers of inflammatory cells including neturophils, eosinophils and $\mathrm{T}$ cells in the lungs following PD-L1 blockade (Supplementary Figure S4A). Taken together, these data demonstrated that PD-L1/PD-1 interaction in the lung is vital to suppress the development of excess pulmonary damage and to promote efficient resolution of pulmonary inflammation. 
We and others have recently showed that IL-10 and Foxp $3^{+}$ Treg cells played important roles in counter-regulating excessive pulmonary inflammation and dampening host diseases during RSV infection. ${ }^{10-16}$ We reasoned that PDL1 blockade might diminish Treg cell infiltration to the lung and/or impair IL-10 production in the airway, thereby leading to enhanced host inflammation and injury during RSV infection. However, PD-L1 blockade resulted in comparable Treg cell infiltration to the lung (Supplementary Figure S4B) and enhanced IL-10 release in the airway during RSV infection (Supplementary Figure S4C). Together, these data suggest that PD-L1 suppresses host diseases independent of the action of Treg and IL-10.

\section{PD-1/PD-L1 interaction controls the inflammatory activities of effector $T$ cells}

We next sought to define the underlying mechanism by which the PD-1 and PD-L1 interaction regulates pulmonary inflammation and injury during RSV infection. As overproduction of inflammatory cytokine by pulmonary effector $\mathrm{T}$ cells contributes significantly to the pulmonary inflammation and host diseases during primary RSV infection, we examined whether PD-L1 affects the in vivo production of inflammatory cytokines by effector T cells during RSV infection. To do so, we injected monensin into RSV-infected mice to block the in vivo release of cytokines by effector $\mathrm{T}$ cells at day 5 post infection, when the peak of inflammatory activities of effector $\mathrm{T}$ cells was observed. ${ }^{16}$ We then stained (intracellular staining (ICS)) lung $\mathrm{T}$ cells for intracellular IFN- $\gamma$ and tumor-necrosis factor- $\alpha$ (TNF- $\alpha$ ) expression directly ex vivo without further stimulation. This in vivo ICS technique allowed us to precisely determine the in vivo production of inflammatory cytokines by effector $\mathrm{CD} 4^{+}$and $\mathrm{CD} 8{ }^{+} \mathrm{T}$ cells (Figure $3 \mathbf{a}$ ). We found that lung effector $\mathrm{T}$ cells isolated from $\alpha$-PD-L1-treated mice displayed a significantly enhanced frequency of both IFN $-\gamma$ and TNF- $\alpha$ producing $\mathrm{CD} 4^{+}$and $\mathrm{CD} 8^{+} \mathrm{T}$ cells compared with those of control mice (Figure 3a,b). The increased frequency of IFN- $\gamma$ - and TNF- $\alpha$-producing cells in vivo following PD-L1 blockade could be caused by increased effector activities of effector T cells or enhanced infiltration and differentiation of RSV-specific T cells into the Th1 or Tc1 lineage. To explore these possibilities, we stimulated the lung effector T cells in vitro with phorbol 12-myristate 13-acetate (PMA) and ionomycin and examined the capabilities of lung effector $\mathrm{T}$ cells to produce IFN- $\gamma$ and TNF- $\alpha$ following mitogenic stimulation. We found that effector $\mathrm{T}$ cells from either control or $\alpha$-PD-L1-treated mice had comparable capability to produce IFN $-\gamma$ and TNF- $\alpha$ in response to mitogenic stimulation (Figure $3 \mathbf{c}, \mathbf{d}$ ), suggesting that PD-L1 blockade at day 4 post infection does not affect the differentiation of $\mathrm{T}$ cells into RSV-specific effector $\mathrm{T}$ cells to the Th1 or Tc1 lineage.

To provide direct evidence that PD-L1 controls the proinflammatory responsiveness of pulmonary effector $\mathrm{T}$ cells during RSV infection, we established an in vitro culture system. Effector $\mathrm{CD}^{+}{ }^{+} \mathrm{T}$ cells were isolated from RSV-infected lungs 8 days post infection (d.p.i.; when spontaneous production of cytokines by effector $\mathrm{T}$ cells due to in vivo antigen $(\mathrm{Ag})$ encounter is minimal ${ }^{16}$ ), and stimulated in vitro with a low concentration of plate-bound $\alpha-\mathrm{CD} 3$ to mimic in vivo $\mathrm{Ag}$ encounter. PD-1 expressed on effector T cells was simultaneously ligated by addition of recombinant PD-L1-Ig fusion protein to the cultures. We found that IFN- $\gamma$ release by effector $\mathrm{T}$ cells was drastically decreased following the ligation of PD-1 (Figure 3e), suggesting that PD-1 ligation on effector T cells directly suppresses the release of IFN- $\gamma$ by effector $\mathrm{T}$ cells. Taken together, these data suggested that PD-L1/PD-1 interaction in the lung suppress the inflammatory activities (release of inflammatory cytokines) of effector $\mathrm{T}$ cells in vivo but not affecting the induction, accumulation or differentiation of lung effector $\mathrm{T}$ cells at this early time point (though it is the peak of in vivo effector T-cell activities) following RSV infection. Consistent with enhanced IL-10 production in the airway, we also found that PD-L1 blockade enhanced IL-10 production by effector $\mathrm{CD}^{+}$and $\mathrm{CD}^{+} \mathrm{T}$ cells (Supplementary Figure S4D,E), again suggesting that the effects of PD-L1 blockade is not due to decreased IL-10 production in vivo during RSV infection.

\section{PD-L1 expression on inflammatory DCs regulates inflammatory activities of effector $T$ cells}

$\mathrm{PD}-\mathrm{L} 1$ is constitutively expressed on various lung cell types and can be further upregulated during infection or inflammation. We next measured PD-L1 expression in the lungs before or during RSV infection. We found that PD-L1 levels in the lung dramatically increased following RSV infection (Figure 4a). Both hematopoietic and non-hematopoietic cells demonstrated increased PD-L1 expression and exhibited the same kinetics as observed in total lung cell suspensions (data not shown).

To determine the major cellular resources of PD-L1 at the time of T-cell infiltration, we examined surface PD-L1 expression by various lung residents and infiltrating cells at day 5 post RSV infection. We found that, although various types of cells in the lung appear to express PD-L1 (Figure 4b), lung inflammatory monocyte-derived DCs $\left(\mathrm{CD} 11 \mathrm{~b}^{+} \mathrm{CD} 11 \mathrm{c}^{+} \mathrm{Ly}_{6 \mathrm{C}^{+}} \mathrm{MHCII}^{+}, 33,34\right.$ Supplementary Figure S5A,B) and lung resident cells such as $\mathrm{CD} 45^{-}$cells (primarily lung epithelial cells) are the two major cellular sources of PD-L1 in the lung during RSV infection (Figure 4c) following adjustment for their levels of PD-L1 expression and relative cellular abundance in the lung (Supplementary Figure S5C). However, inflammatory DCs appear to be the dominant cellular source of PD-L1 (Figure 4c). We also found that inflammatory DC cell numbers in the lung increased drastically following RSV infection (Supplementary Figure S5D), and PD-L1 expression on inflammatory DCs increased similarly to expression in total lung cells following RSV infection (Supplementary Figure S5E).

During acute influenza infection, inflammatory DCs, but not lung resident cells, have been reported to predominantly stimulate inflammatory cytokine release by effector $\mathrm{T}$ cells in the lungs. ${ }^{35}$ We therefore hypothesized that PD-L1 expressed by inflammatory DCs, rather than by $\mathrm{CD} 45^{-}$lung resident cells, serves to control excessive inflammatory activities of 

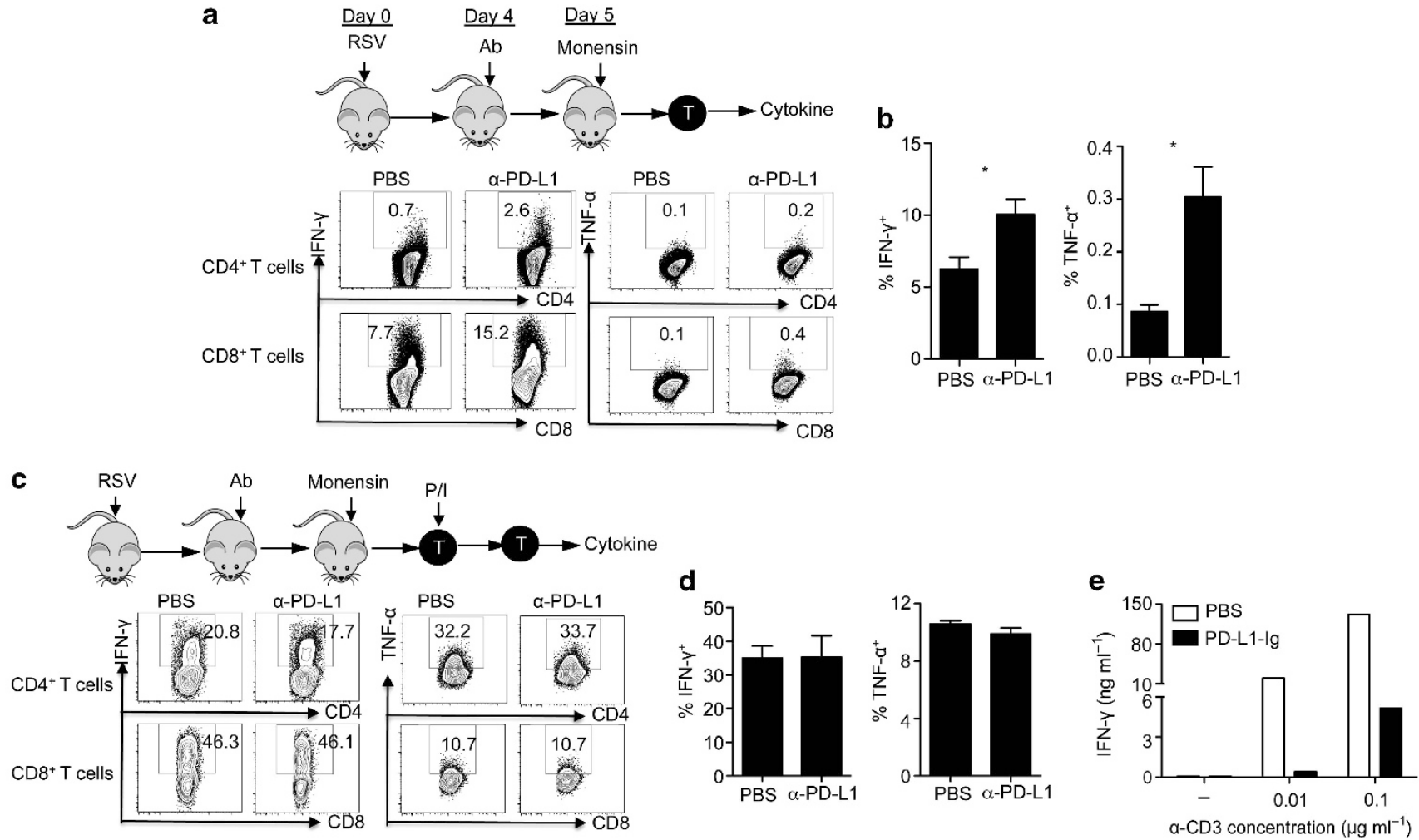

Figure 3 PD-L1 blockade results in enhanced inflammatory activities of effector T cells. (a-d) Wild-type Balb/c mice were infected with respiratory syncytial virus (RSV) and treated with phosphate-buffered saline (PBS) or $\alpha$-PD-L1. At day 5 post infection, mice were injected with monensin to block the in vivo secretion of cytokines by T cells. (A) Direct interferon- $\gamma$ (IFN- $\gamma$ ) and tumor-necrosis factor- $\alpha$ (TNF- $\alpha$ ) staining of ex vivo lung CD4 ${ }^{+}$and CD8 ${ }^{+}$T $^{-}$

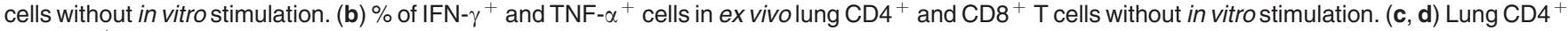
and $\mathrm{CD}^{+} \mathrm{T}$ cells were stimulated with phorbol 12-myristate 13-acetate (PMA) and lonomycin (c). IFN- $\gamma$ and TNF- $\alpha$ production by T cells are depicted. (d) $\%$ of IFN $-\gamma^{+}$and TNF- $\alpha^{+}$cells in lung CD4 ${ }^{+}$and CD8 ${ }^{+} \mathrm{T}$ cells following PMA and lonomycin stimulation. (e) Effector CD8 ${ }^{+} \mathrm{T}_{\text {cells were isolated from }}$ RSV-infected lungs (day 8 ) and stimulated in vitro with increasing amount of plate-bound $\alpha$-CD3 $\left(0.01\right.$ and $\left.0.1 \mu \mathrm{g} \mathrm{ml}^{-1}\right)$ in the absence or presence of recombinant PD-L1-Ig protein. IFN- $\gamma$ concentrations in the supernatant following $24 \mathrm{~h}$ stimulation are depicted. Data are representative of three independent experiments. ${ }^{*}, P<0.05$ as determined by unpaired Student's $t$-test.

effector T cells during RSV infection. To test this idea, we cocultured either inflammatory DCs or lung resident cells with $\mathrm{CD}^{+}$effector T cells isolated from RSV-infected lungs, and measured production of IFN- $\gamma$ in supernatant in the absence or presence of $\alpha$-PD-L1. Consistent with findings in the influenza infection, ${ }^{35}$ we found that only minimal amount of IFN- $\gamma$ was produced in the lung resident cell-T co-culture, while copious amount of IFN- $\gamma$ was detected in inflammatory DC-T coculture (Figure 4d), indicating that inflammatory DCs, rather than the lung resident cells, are the primary Ag presenting cells in vivo to stimulate cytokine production by effector $\mathrm{T}$ cells during RSV infection. Strikingly, blockade of the PD-L1/PD-1 interaction between inflammatory DCs and effector $\mathrm{CD} 8^{+} \mathrm{T}$ cells resulted in greatly enhanced IFN $-\gamma$ production by effector $\mathrm{CD}^{+} \mathrm{T}$ cells (Figure 4d). Thus, our data indicated that PD-L1 expressed by inflammatory DCs controls inflammatory activities of effector $\mathrm{T}$ cells in the lung in vivo during RSV infection.

\section{An IFN-STAT1-IRF1 axis is critical to induce PD-L1 expression on inflammatory DC during RSV infection}

We next sought to determine the molecular mechanism by which PD-L1 is induced on inflammatory DCs during RSV infection. The induction of PD-L1 on inflammatory DCs during RSV infection is independent of IL-10 since IL-10R blockade did not affect PD-L1 expression on inflammatory DCs (Supplementary Figure S5F). IFNs, in particular IFN- $\gamma$, are capable of inducing PD-L1 expression on DCs and macrophages in vitro and in vivo. ${ }^{36,37}$ We next examined whether STAT1, the IFN-downstream signaling molecule ${ }^{38}$ is required for the expression of PD-L1 on inflammatory DCs during RSV infection. Since STAT1 is an important innate antiviral molecule, to avoid potential additional complexity due to the effects of STAT1 deficiency on epithelial anti-viral responses, we generated a wild-type (WT) and STAT1deficient mixed bone marrow (BM) chimera. To do so, we lethally irradiated WT mice and then reconstituted the mice with mixed WT and STAT1-deficient BM cells (1:1 ratio). Twelve weeks after reconstitution, we generated chimeric mice that have WT epithelial cells with 1:1 mixed WT or STAT1deficient hematopoietic cells (Figure 5a). We then infected the mice with RSV and examined PD-L1 expression on lung inflammatory DCs at day 5 post infection. We found that STAT1 deficiency drastically diminished PD-L1 levels on inflammatory DCs (Figure 5b), suggesting that intrinsic STAT1 signaling in inflammatory DCs is required for the 
a

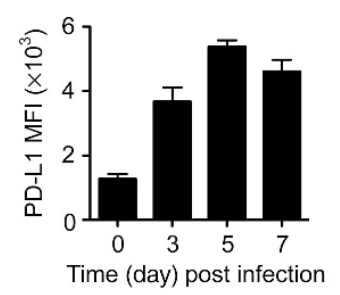

C

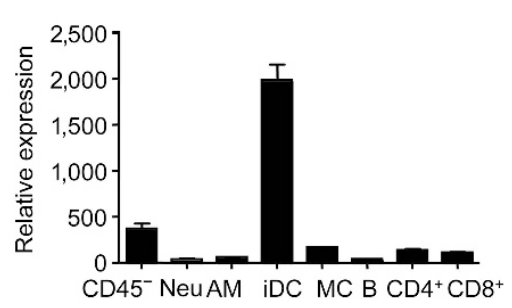

b

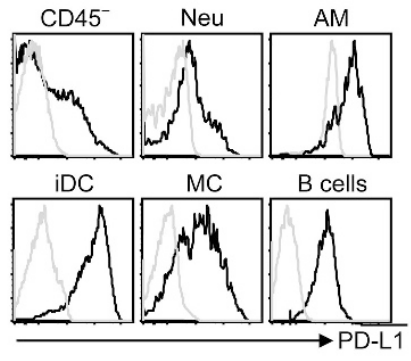

d
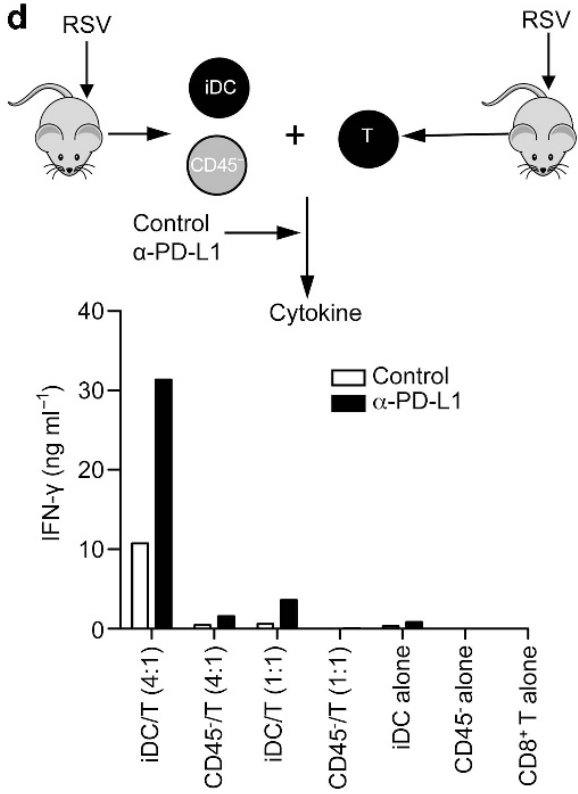

Figure 4 Inflammatory dendritic cell (DC)-derived PD-L1 inhibits cytokine production by effector T cells. (a-c) Wild-type Balb/c mice were infected with respiratory syncytial virus (RSV). (a) PD-L1 expression (mean fluorescence intensity (MFI)) in the lung cells. (b) PD-L1 expression on various cell types in

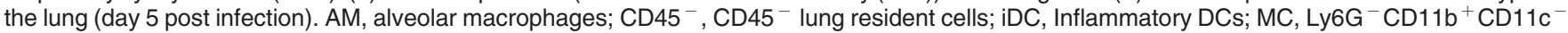
monocytes; Neu, neutrophils. (c) Relative expression levels of PD-L1 on different cell types (PD-L1 relative expression was calculated by PD-L1 MFI $\times$ \% of cells in the lungs). (d) iDC and CD45- resident cells were sorted from the infected lungs at day 5 post infection. Cells were then mixed with day 8 lung $\mathrm{CD}^{+}$effector T cells in the presence of control Ab or $\alpha$-PD-L1. Interferon- $\gamma$ concentrations in the supernatant following $24 \mathrm{~h}$ stimulation are depicted. Data are representative of two to three independent experiments.

expression of PD-L1. We next examined whether type I IFNs $($ IFN- $\alpha / \beta)$ or II IFN (IFN- $\gamma$ ) is required for the induction of PDL1 on inflammatory DCs. We found that inflammatory DCs from IFNAR1-deficient mice or from mice in which IFN- $\gamma$ has been neutralized exhibited slightly diminished PD-L1 expression, demonstrating that type I or II IFN-deficiency alone only moderately decreased PD-L1 expression (Figure 5c,d). Strikingly, the co-blockade of type I and II IFNs dramatically inhibited the expression of PD-L1 on inflammatory DCs (Figure 5e). The blockade of type I and II IFNs together also diminished PD-L1 messenger RNA in inflammatory DCs (Figure 5f). Taken together, these data suggested that PD-L1 expression on inflammatory DCs in vivo during RSV infection requires the co-ordination of type I and II IFN signaling through STAT1. Consistent with this idea, we found that type I and II IFNs co-operated to enhance PD-L1 expression on BM-derived DCs (BMDCs) in vitro (Figure 5g).

We next investigated transcription factors downstream of IFN/STAT1 that are required for the expression of PD-L1 on inflammatory DCs. To this end, we found that both type I and II IFNs enhanced expression of the transcription factor IRF1 in a manner dependent on STAT1 (Figure 6a). IRF1 expression was previously shown to be required for PD-L1 expression on human cancer cell lines in vitro. ${ }^{39}$ However, the role of IRF1 in regulating $\mathrm{PD}-\mathrm{L} 1$ expression in other cell types, especially in the in vivo setting was not previously investigated. We therefore investigated the role of IRF1 in regulating PD-L1 expression on inflammatory DCs. Consistent with our in vitro data, we found the absence of type I and II IFNs and IFN receptor signaling in vivo diminished IRF1 expression in inflammatory DCs (Supplementary Figure S6). To examine the role of IRF1 in regulating $\mathrm{PD}-\mathrm{L} 1$ expression on inflammatory DCs in vivo, we infected IRF1-deficient mice with RSV and examined PD-L1 expression on lung inflammatory DCs at day 5 post infection. We found that IRF1 deficiency significantly impaired PD-L1 expression on lung inflammatory DCs in vivo (Figure $\mathbf{6 b}, \mathbf{c}$ ). IRF1 is also an important anti-viral molecule and IRF1 is required for $\mathrm{CD}^{+}{ }^{+}$T-cell development. ${ }^{40}$ To avoid these 


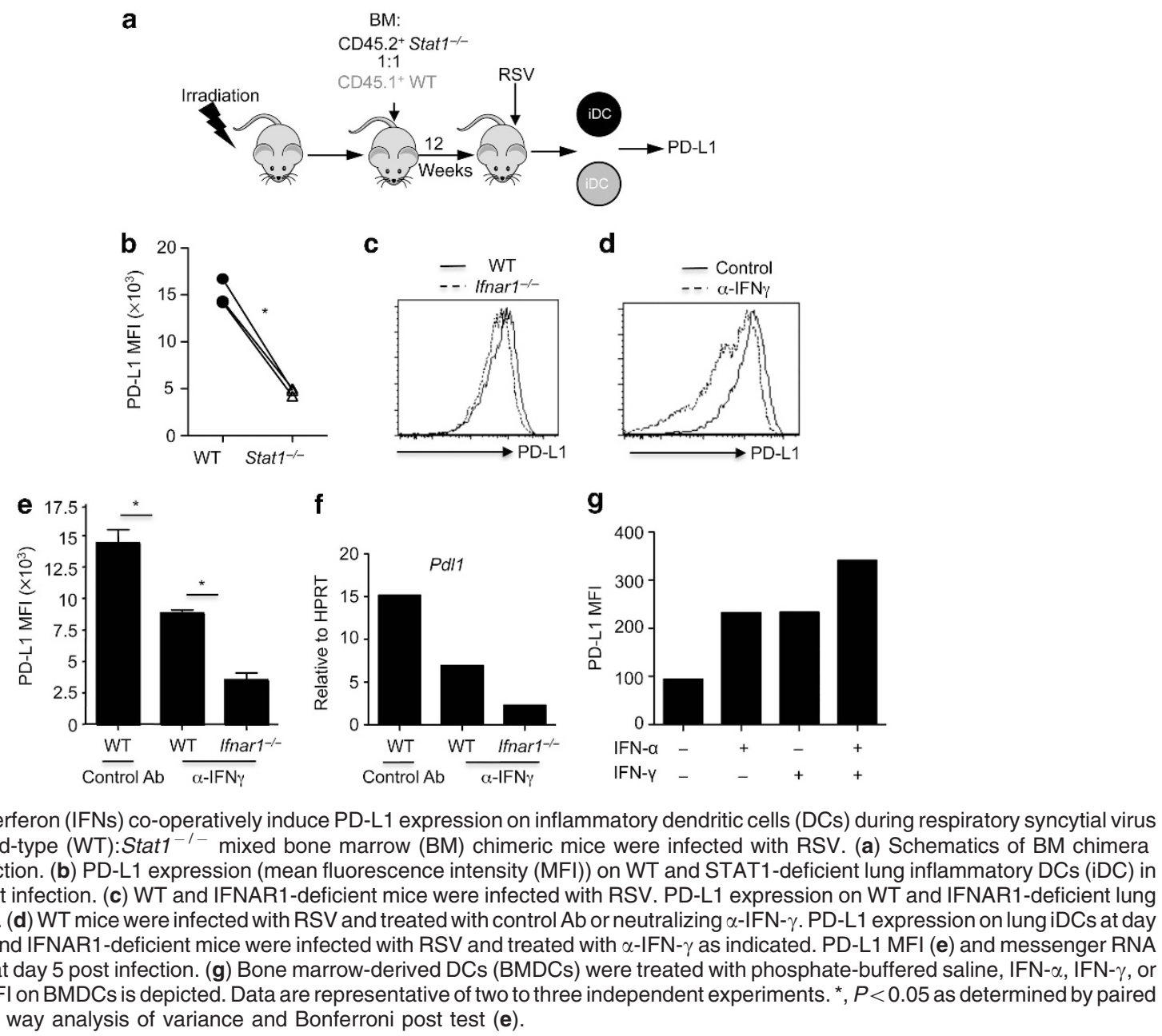

Figure 5 Type I and II interferon (IFNs) co-operatively induce PD-L1 expression on inflammatory dendritic cells (DCs) during respiratory syncytial virus (RSV) infection. (a, b) Wild-type (WT):Stat $1^{-1-}$ mixed bone marrow (BM) chimeric mice were infected with RSV. (a) Schematics of BM chimera construction and RSV infection. (b) PD-L1 expression (mean fluorescence intensity (MFI)) on WT and STAT1-deficient lung inflammatory DCs (iDC) in the same host at day 5 post infection. (c) WT and IFNAR1-deficient mice were infected with RSV. PD-L1 expression on WT and IFNAR1-deficient lung iDCs at day 5 post infection. (d) WT mice were infected with RSV and treated with control Ab or neutralizing $\alpha$-IFN- $\gamma$. PD-L1 expression on lung iDCs at day 5 post infection. (e, f) WT and IFNAR1-deficient mice were infected with RSV and treated with $\alpha-I F N-\gamma$ as indicated. PD-L1 MFI (e) and messenger RNA message (f) on lung iDCs at day 5 post infection. (g) Bone marrow-derived DCs (BMDCs) were treated with phosphate-buffered saline, IFN- $\alpha$, IFN- $\gamma$, or IFN- $\alpha$ plus IFN- $\gamma$. PD-L1 MFI on BMDCs is depicted. Data are representative of two to three independent experiments. ${ }^{*}, P<0.05$ as determined by paired Student's $t$-test $(\mathbf{b})$ or one way analysis of variance and Bonferroni post test (e).

potential caveats, we generated mixed bone marrow chimera (Figure 6d) as described above (Figure 5). We found that IRF1-deficient inflammatory DCs exhibited impaired PD-L1 expression compared with their WT counterparts under the same environment (Figure 6e). These data collectively demonstrated that intrinsic expression of IRF1 by inflammatory DCs is required for the expression of PD-L1 on inflammatory DCs in vivo during RSV infection. To examine whether IRF1 expression is sufficient to promote PD-L1 expression on DCs, we transduced BMDCs with control or IRF1-expressing retroviruses and examined PD-L1 expression on transduced DCs. We found that ectopic IRF1 expression enhanced PD-L1 expression on BMDCs (Figure $\mathbf{6 f}, \mathbf{g}$ ), suggesting that IRF1 is sufficient to induce PD-L1 expression on DCs. Since IRF1 was required for PD-L1 expression and PD-L1 expression on inflammatory DCs controlled inflammatory activities of effector T cells, we reasoned that IRF1 deficiency in inflammatory DCs would augment cytokine production by effector T cells upon Ag encounter. To test this hypothesis, we infected WT or IRF1-deficient mice with RSV, isolated WT or IRF1deficient inflammatory DCs and then mixed them with WT effector T cells from RSV-infected lungs. We found that IRF1 deficiency in inflammatory DCs, (but not in CD $45^{-}$epithelial cells), greatly augmented IFN- $\gamma$ production by effector $\mathrm{T}$ cells (Figure 6h). Taken together, these data suggested that an IFN-STAT1-IRF1 axis is required for the expression of PD-L1 on inflammatory DCs and the control of inflammatory activities of effector T cells during RSV infection.

\section{DISCUSSION}

In this report, we have examined the expression and function of PD-1 and PD-L1, specifically in the lung, during RSV infection. We found that PD-1 and PD-L1 interactions minimally affected RSV viral clearance but drastically modulated the development of excessive pulmonary inflammation and lung injury during RSV infection. We found that engagement of PD1 by inflammatory DC-derived PD-L1 is critical for the regulation of pro-inflammatory cytokine release by effector $\mathrm{CD}^{+}$and $\mathrm{CD}^{+}{ }^{+} \mathrm{T}$ cells, and as a result, the control of the inflammatory effector T-cell activities in vivo in the lung. In addition, we have identified the physiological cues and underlying signaling pathways responsible for PD-L1 expression on inflammatory DCs during RSV infection. We found that an IFN-STAT1-IRF1 axis is critical for the upregulation of 
a

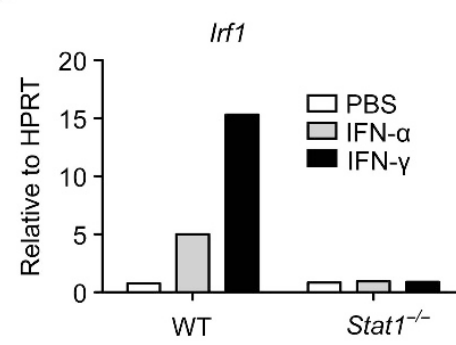

d

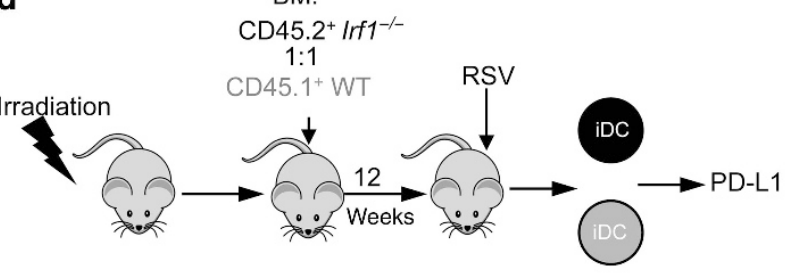

b - Isotype
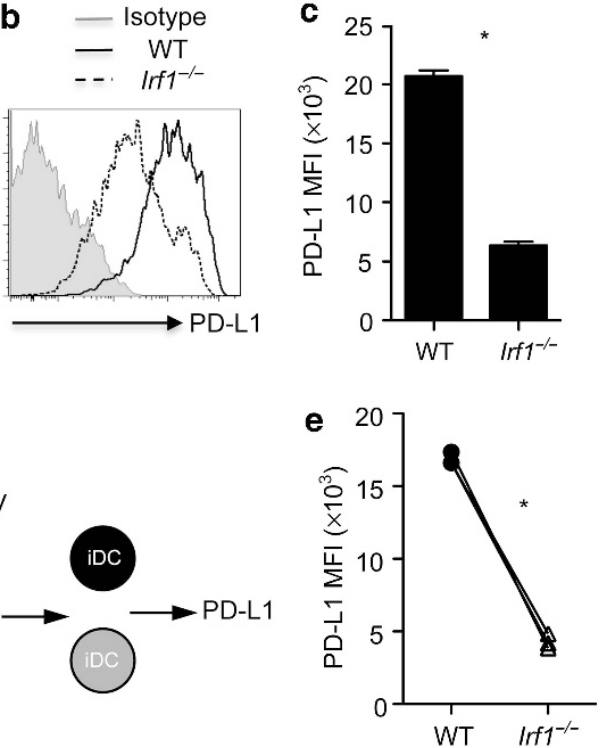

f

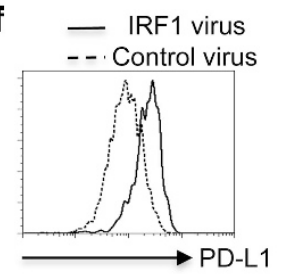

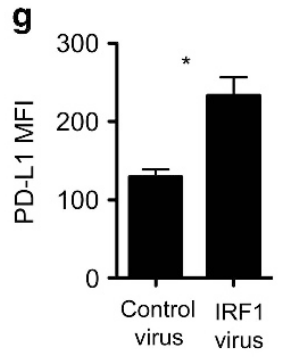

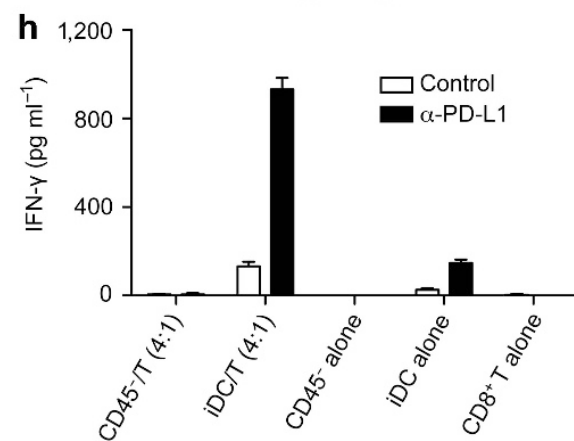

Figure 6 IRF1 is required for PD-L1 expression on inflammatory DCs (iDC) during respiratory syncytial virus (RSV) infection. (a) IRF1 expression in wild-type (WT) or STAT1-deficient bone marrow-derived DCs (BMDCs) treated with phosphate-buffered saline (PBS), interferon- $\alpha$ (IFN- $\alpha$ ) or IFN- $\gamma$. (b, c) PD-L1 expression (b) and mean fluorescence intensity (MFI) (c) on iDCs isolated from lungs of RSV-infected WT or IRF1-deficient mice (day 5 post infection). (d, e) WT: Irf1 ${ }^{-1}$ - mixed BM chimeric mice were infected with RSV. (d) Schematics of BM chimera construction and RSV infection. (e) PD-L1 expression (MFI) on WT and IRF1-deficient lung iDCs in the same host at day 5 post infection. (f, $\mathbf{g})$ BMDCs were transduced with control or IRF1expressing retroviruses. PD-L1 expression (f) and MFI (g) on control virus or IRF1-expressing-virus transduced DCs are depicted. (h) WT or IRF1deficient lung iDCs was sorted from infected WT or Irf $1^{-1}$ mice, respectively, at day 5 post infection. Cells were then mixed with day 8 lung CD8 ${ }^{+}$effector $\mathrm{T}$ cells in the presence of control Ab or $\alpha$-PD-L1. IFN- $\gamma$ concentrations in the supernatant following $24 \mathrm{~h}$ stimulation are depicted. Data are representative of three independent experiments. ${ }^{*}, P<0.05$ as determined by unpaired $(\mathbf{c}, \mathbf{g})$ or paired Student's $t$-test $(\mathbf{e})$.

PD-L1 on inflammatory DCs and as a consequence, suppression of the release of cytokine production by effector T cells.

We found that PD-1 is highly expressed on both human and mouse $\mathrm{T}$ cells at the respiratory tract during RSV infection. Currently, due to the extreme low numbers of cells we recovered from the nasal washes of healthy children, we were unable to determine whether PD-1 is expressed on human CD4 and/or CD8 T cells residing in the respiratory tract of healthy children. However, we found that PD- 1 is minimally expressed on T cells in the respiratory tract in uninfected mice, suggesting that local inflammation and/or Ag encounter at the respiratory tract trigger the upregulation of PD-1 on T cells during RSV infection. These data are in line with recent reports of PD-1 expression on lung $\mathrm{T}$ cells during influenza and HMPV infection. ${ }^{24-26}$ Interestingly, we further found that PD-1 is highly upregulated on IL-10 producing effector $\mathrm{T}$ cells, suggesting that $\mathrm{IL}-10$ and $\mathrm{PD}-1$ expression in $\mathrm{T}$ cells may be regulated by the same molecular machinery during RSV infection. Notably, we recently showed that the transcription factor Blimp-1 promoted IL-10 production by effector T cells during influenza infection. Furthermore, Blimp-1 modulates $\mathrm{PD}-1$ expression during chronic virus infection. ${ }^{41}$ Thus, Blimp1 may promote the upregulation of PD-1 on effector T cells in the respiratory tract, although further studies are warranted. We also detected significant expression of inhibitory molecules such as Lag-3 and Tim-3 on IL-10 producing effector T cells (data not shown), suggesting that certain lung effector $\mathrm{T}$ cells simultaneously express multiple inhibitory molecules along with the regulatory cytokine IL-10. We believe that such a regulatory constellation may reflect the process of lung effector $\mathrm{T}$ cells adapting to the local inflammatory environment to restrict excess production of inflammatory mediators that enhance lung injury. Interestingly, it was shown recently that $\mathrm{PD}$-1-expressing exhausted $\mathrm{T}$ cells undergo a form of 
differentiation that can be stably transmitted to daughter cells during chronic lymphocytic choriomeningitis virus infection. ${ }^{42}$ It is thus enticing to speculate that these PD-1-expressing effector $\mathrm{T}$ cells at sites of infection/inflammation may also pass through a stable and transmissible differentiation state. Furthermore, we observed that PD-1 expression on human airway $\mathrm{T}$ cells was quite variable among different individuals. Given the limited numbers of human samples in our study, we were unable to correlate PD-1 expression on airway T cells with the severity of host diseases. Future large-scale studies are warranted for the determination of whether PD-1 expression levels and/or polymorphisms in PD-1 locus are associated with the severity of RSV-induced diseases in children. Of note, a polymorphism in the locus of IL-10, another important immune-regulatory factors in restricting host inflammatory responses during murine RSV infection, ${ }^{14-16}$ was shown to be associated with the severity of bronchiolitis in infants infected with RSV. ${ }^{17,18,43}$

PD-1 interacts with both PD-L1 and PD-L2. ${ }^{19}$ We found that the blockade of PD-L1 but not PD-L2 greatly enhanced host morbidity and lung inflammation/injury during RSV infection. The different expression levels of PD-L1 and PD-L2 in the lung following RSV infection may explain the differential function of PD-L1 and PD-L2 on host disease development. Notably, PDL2 expression is primarily controlled by type 2 cytokines such as IL-4 and IL-13. ${ }^{44}$ Unlike RSV A2 strains used in this study, certain RSV strains, including line19 and 2-20, were shown to selectively induce Th2 cytokine production and asthma-like symptoms including mucus production and airway resistance. ${ }^{45,46}$ Therefore, it is possible that PD-L1 and PD-L2 blockade may have different functional consequences during the infections by different RSV strains. Of note, the blockade of PD-L2 was reported to selectively enhance Th2 cytokine production and asthmatic symptoms in a murine model of asthma. ${ }^{44}$

The treatment with blocking anti-PD-L1 antibody during HMPV and influenza infections has been demonstrated to greatly enhance $\mathrm{CD}^{+} \mathrm{T}$ cell numbers and in the case of influenza facilitate viral clearance. ${ }^{24-26}$ However, only modest effects on host morbidity and lung injury were noted. ${ }^{24-26}$ In contrast, we showed here that the PD-L1 blockade only modestly affected the early anti-RSV T cell response and viral clearance but drastically promoted host weight loss and tissue injury. Such dichotomy may be attributed to the inherent differences in virus replication, tissue tropism, and the immune response stimulated between RSV, HMPV, and influenza. During the infection with viruses that replicate to high levels such as influenza and HMPV infection, the beneficial effect of PD-L1 in restricting exuberant T-cell-mediated inflammation is probably compensated by enhanced virus induced injury caused by the reduction of T cell anti-viral activity. Furthermore, previous studies blocked PD-L1 activity prior to infection. ${ }^{24-26}$ Since PD-1 can be transiently upregulated during $\mathrm{T}$ cell priming, ${ }^{19,28}$ it is possible PD-L1 blockade may enhance T-cell activation and proliferation during $\mathrm{T}$ cell priming within the draining lymph nodes, thereby promoting effector $\mathrm{T}$ cell accumulation and function at the site of infection. In contrast, we chose to block PD-L1 and PD-1 interaction at the time of $\mathrm{T}$ cell infiltration to the lung to specifically determine the function of PD-L1 and PD-1 interaction in the lung in regulating tissue inflammation and viral clearance during RSV infection. Our results showed that acute PD-L1 blockade minimally affected effector T-cell priming and $\mathrm{Th} 1 / \mathrm{Tcl}$ differentiation as determined by the in vitro ICS staining at day 5 post infection. However, as determined by in vivo ICS, acute blockade of PD-L1 drastically enhanced in vivo production of the inflammatory cytokines, IFN- $\gamma$, and TNF- $\alpha$, which were previously shown to contribute to host morbidity and tissue injury during RSV infection. ${ }^{47,48}$ These data suggest that the primary function of PD-L1 and PD-1 interaction in the infected lungs in vivo is to limit inflammatory activities of $\mathrm{T}$ cells in situ upon their arrival at the site of infection (i.e. the lungs) and, thereby suppress the development of excessive pulmonary inflammation and injury during RSV infection. Notably in this regard, the blockade of PD-L1 in vivo lead to enhanced accumulation of effector T cells in the lung later during RSV infection (i.e. day 9), presumably due to enhanced recruitment caused by enhanced chemokine levels in the lung and/or elevated local proliferation/survival of effector $\mathrm{T}$ cells in the lung. Furthermore, although we did not directly examine Th2 and Th17 cytokine production by T cells during RSV infection, we believe that the acute blockade of PDL1 did not alter the nature of inflammatory type, but rather resulted in a much intensified inflammatory immune response as evidenced by the increased levels of Th1, Th2, and Th17 associated cytokines present in the airway of mice that received $\alpha$-PD-L1.

We previously demonstrated that lung $\mathrm{CD}_{11 \mathrm{c}^{+}}$cells (mainly inflammatory DCs) are required to stimulate the in situ production of effector cytokines during influenza infection. ${ }^{35}$ Similarly, we found that lung inflammatory DCs rather than $\mathrm{CD} 45^{-}$cells (primarily epithelial cells) potently stimulated cytokine production by effector $\mathrm{T}$ cells during RSV infection, in spite of the fact that RSV primarily infects epithelial cells. Previous analyses have shown that differential activities of effector $\mathrm{T}$ cells (i.e., cytokine production vs. killing of target cells) are governed by the strength of stimulation they received following interaction with target cells. ${ }^{35,49,50}$ The engagement of co-stimulatory receptors (i.e., B7 molecules) on effector $\mathrm{CD}^{+}{ }^{+} \mathrm{T}$ cells by lung $\mathrm{CD} 11 \mathrm{c}^{+}$DCs provides the additional signal strength necessary for the production of proinflammatory cytokines by effector $\mathrm{T}$ cells, while lung epithelial cells, which lack the expression of B7 molecules, deliver weak stimulation signals to effector $\mathrm{T}$ cells adequate for the triggering of the T-cell killing degranulation process but not effector cytokine production. ${ }^{35,49}$ We showed here that lung CD11c $\mathrm{c}^{+}$ inflammatory DCs also express high levels of PD-L1 and thus are capable of delivering negative signals to effector $\mathrm{T}$ cells to limit their cytokine production. Thus, DCs possess both positive and negative signals to modulate the in situ inflammatory activities of tissue effector $\mathrm{T}$ cells and thereby directly impact viral pathogenesis through modulation of 
effector $\mathrm{T}$ cells. Interestingly, as we reported previously following IL-10 receptor blockade, the down-modulation of the inflammatory activities of effector $T$ cells does not significantly alter the ability of effector $\mathrm{T}$ cells to aid in virus clearance, suggesting that it is possible to uncouple inflammatory activities of effector T cells and their anti-viral activities in vivo during RSV infection. Such strategies, achieved by either selective blockade of co-stimulatory signaling in situ, such as CD28 signaling, or by enhancing the activities of inhibitory molecule activities (such as IL-10 and PD-L1, or Treg activities) may serve as potential future therapeutics for acute respiratory viral infections.

Both type I and II IFNs have been shown to induce PD-L1 expression on DCs in vitro. However, the relative contributions of these cytokines in the in vivo expression of PD-L1 are less clear. Through the blockade of the action of type I and/or type II IFNs, we showed that type I and II IFNs co-operatively induced the expression of PD-L1 in vivo. Interestingly, different strains of RSV viruses have been shown to differentially regulate type I and/or II IFN production in vivo, ${ }^{51}$ suggesting that they may also differentially regulate PD-L1 expression in vivo. Nevertheless, our finding that PD-L1 blockade leads to enhanced IFN- $\gamma$ production in vivo, suggesting that pro-inflammatory IFN- $\gamma$ response of effector $\mathrm{T}$ cells in response to RSV infection is regulated through a feedback loop mediated by the regulated expression of PD-L1 on DCs. Mechanistically, both type I and II IFNs induced IRF1 gene expression in DCs. IRF1 was previously demonstrated to regulate PD-L1 expression on human tumor cell lines. ${ }^{39}$ We found that ectopic expression of IRF1 induced PD-L1 expression on DCs and the ablation of IRF1 in vivo abrogated PD-L1 expression. Thus, these multiple lines of evidence described here formally established that IRF1 is critical for the expression of PD-L1 on DCs in vivo during RSV infection.

In summary, we showed that PD-1 is highly expressed on effector $\mathrm{T}$ cells in the respiratory tract during both murine and human RSV infection. We demonstrated that PD-L1-PD-1 interaction is critically important for restricting the inflammatory activities of effector $\mathrm{T}$ cells during RSV infection. Furthermore, we identified that the IFN-STAT1-IRF1 molecular axis in inflammatory DCs is critical for the physiological regulation of PD-L1 and the in situ activities of effector T cells in the lungs. Our findings may provide the groundwork for future studies employing PD-1-PD-L1 pathway as a therapeutic option in the treatment of severe RSV infection.

\section{METHODS}

Ethics statement. Patient sample collection was approved by Indiana University (IU) Institutional Review Boards (IRB, IRB approval number: 1212010151). Written informed consents were signed by English-speaking parents or guardians. Animal study was carried out in strict accordance with the Animal Welfare Act (Public Law 91-579) and the recommendations in the Guide for the Care and Use of Laboratory Animals of the National Institutes of Health (OLAW/NIH, 2002). The protocol was approved by the University of Virginia Animal Care and Use Committee (UVA ACUC, Protocol Number: 2230) and the animal care and use program at Indiana University School of Medicine (IUSM, Protocol number: 10006). Both institutions are in compliance with all applicable federal regulations and accredited by AAALAC. Daily care for the animals was provided by the trained staff from Laboratory Animal Resource Center (LARC) in UVA and IUSM.

RSV patients and healthy controls. A total of $10 \mathrm{RSV}$ subjects and 4 healthy controls were selected for this study. Nasal washes from six subjects contained sufficient numbers of cells and were analyzed and compared with cells from peripheral blood mononuclear cells from these subjects. We were unable to obtain enough cell numbers in nasal washes for flow cytometry analysis in healthy patients and the other RSV subjects. All RSV subjects and healthy controls were recruited during the 2013 RSV season. The chosen RSV subjects were inpatients at Riley Hospital for Children in Indianapolis, and confirmed to be RSV positive by direct fluorescent antibody assay or PCR. Healthy controls were included if no viral infection, fever, and usage of antibiotic were detected. All selected RSV subjects and healthy controls were $<3$ years of age without diagnosis of chronic diseases, bleeding disorder, history or current use of palivizumab, and inability to provide blood sample; and have to be accompanied by English-speaking parents or guardians.

Nasal wash procedure. The subject was placed on a continuous oxygen monitor for this procedure. During the procedure, the subject was held in the lateral recumbent position, and $5 \mathrm{ml}$ of sterile normal saline solution was instilled into the upper nostril 1-2 $\mathrm{ml}$ at a time over a total time of 30-60 s. Nasal lavage fluid (NLF) passively flowing from the inferior nostril was collected in a cup or specimen container. A soft rubber bulb syringe was used to gently suction any remaining fluid from both nostrils and added to the material in the cup. The NLF was then transferred to a capped polypropylene tube, placed on crushed ice, and then transported to the laboratory for initial processing within $2 \mathrm{~h}$ of the lavage being completed.

Blood draw. Five milliliters (minimum of $3 \mathrm{ml}$ ) of whole blood was obtained by peripheral venipuncture. For the patient who already has a central venous catheter in place, blood was drawn from the central venous catheter preferentially. After the blood was drawn, the sample was transferred immediately to the laboratory for initial processing.

Collection of cells from blood and NLF. Peripheral blood mononuclear cells were isolated over Ficoll; for nasal wash samples, the fluid was first filtered over a $70-\mu \mathrm{m}$ cell strainer (BD Falcon, Bedford, MA), and then centrifuged for $5 \mathrm{~min}$ at 1,500 r.p.m. at $4{ }^{\circ} \mathrm{C}$. Both the cell pellets from blood and NLF were re-suspended in $20 \%$ fetal bovine serum-RPMI (Gibco, Grand Island, NY) supplemented with 10\% dimethylsulfoxide, and immediately frozen for future staining.

Mice and infection. WT BALB/c and C57BL/6 mice were purchased from The Jackson Laboratory (Bar Harbor, ME). IL-10-enhancedgreen fluorescent protein reporter mice (Vert-X), Ifnar $1^{-1-}$, Stat $1^{-1-}$, and $\operatorname{Irf1} 1^{-1-}$ mice were bred in house. All mice were housed in a specific pathogen-free environment and all animal experiments were performed in accordance with protocols. The A2 strain of RSV was grown in HEp-2 cells (ATCC, Manassas, VA) and tittered for infectivity. Mice were infected at age of 8-10 week with a dose of $1-1.2 \times 10^{7}$ p.f.u. RSV in serum-free Iscove's medium (Gibco) intranasally following anesthesia with ketamine and xylazine.

Administration of $\mathbf{A b}$ in vivo. Mouse PD-L1 (B7-H1) monoclonal antibody (clone 10B5) was purified on a protein G column from the supernatant of the hybridoma cell line (kindly provided by Dr Lieping Chen from Yale University). Anti-PD-L2 blocking Ab (clone TY25) was purchased from Bio-X-Cell (West Lebanon, NH). Control Abs (hamster IgG and Rat IgG) were obtained from Jackson ImmunoResearch Laboratories. The blocking Abs were injected IP with a dose of $1.2 \mathrm{mg}$ per mouse at 4 d.p.i. and $600 \mu \mathrm{g}$ per mouse at 6 d.p.i., respectively. Neutralizing IFN- $\gamma$ Ab (clone XMG1.2, Bio-X-Cell) was injected IP at dose of $1 \mathrm{mg}$ per mouse. 
Evans-blue dye leakage measuring lung injury. Twenty-four hours following the secondary anti-PD-L1 blockade Ab injection ( 7 days post RSV infection), mice were injected via the tail vein with Evans-Blue Dye $\left(50 \mathrm{mg} \mathrm{kg}^{-1}\right)$. Thirty minutes following the injection, BAL was performed by flushing the airway multiple times with a single volume of $600 \mu \mathrm{l}$ sterile phosphate-buffered saline. BAL fluid was then spun down and the supernatant was collected to measure the absorbance at $620 \mathrm{~nm}$ by spectrophotometer.

Histology. Twenty-four hours post the secondary anti-PD-L1 or control IgG Ab injection on day 7 post infection, whole lungs of RSVinfected mice were harvested and fixed in $10 \%$ buffered formalin phosphate (Fisher Scientific, Fair Lawn, NJ). Fixed lungs were embedded in paraffin, sectioned at $4-\mu \mathrm{m}$ thickness, and stained by the Indiana University Pathology Laboratory. Slides were then stained with hematoxylin and eosin.

Airway cytokine determination. BAL fluid with a volume of $600 \mu \mathrm{l}$ was obtained and spun down as described. ${ }^{52}$ Supernatants were then collected for multiplex analysis (Millipore, Billerica, MA) according to the manufacturer's instructions.

Ex vivo restimulation of pulmonary effector $\mathrm{CD}^{+} \mathrm{T}$ cells. Pulmonary effector $\mathrm{CD} 8^{+}$T cells were purified from RSV-infected lungs 8 d.p.i. using MACS-beads (Miltnenyi Biotec) and seeded into round-bottom 96 wells $\left(10^{5}\right.$ cells per well) in presence of plate-bound $\alpha-\mathrm{CD} 3 \mathrm{Ab}$ with various concentrations to trigger TCR engagement, inducing robust inflammatory cytokine production by lung effector $\mathrm{T}$ cells. Recombinant PD-L1-Ig fusion protein $(\mathrm{R} \& \mathrm{D})$ was added at the time of $\alpha-\mathrm{CD} 3 \mathrm{Ab}$ administration with a concentration of $10 \mu \mathrm{g} \mathrm{ml}^{-1}$ to interact with PD-1 expressed at effector T cells. CD8 ${ }^{+} \mathrm{T}$ cells cultured with medium alone with/without PD-L1-Ig were served as controls. Supernatants were harvested $24 \mathrm{~h}$ after co-incubation and enzyme-linked immunosorbent assay (ELISA) was performed to measure the inflammatory cytokine IFN- $\gamma$ production.

In co-culture of $\mathrm{CD} 8{ }^{+} \mathrm{T}$ cells with inflammatory DCs/epithelial cells, effector CD8 ${ }^{+} \mathrm{T}$ cells were purified from RSV-infected lungs 8 d.p.i. and seeded into 96-well round-bottom wells with a concentration of $5 \times 10^{4}$ cells per well. Inflammatory DCs and epithelial cells (CD45-) were directly sorted from RSV-infected lungs 5 d.p.i. and co-incubated directly with $\mathrm{CD} 8{ }^{+} \mathrm{T}$ cells at various ratios. $\alpha$-PD-L1 was finally added into the co-culture with a concentration of $20 \mu \mathrm{g} \mathrm{ml}^{-1}$ to block the interaction between PD-L1 (expressed by inflammatory DCs/epithelial cells) and PD-1 (expressed by CD $8^{+}$T cells). Twenty-four hours later, supernatants were harvested to measure the production of IFN- $\gamma$ by ELISA (Biolegend, San Diego, CA).

Quantitative reverse transcription PCR. RNA from the infected lungs was isolated via Trizol (Invitrogen, Grand Island, NY) and treated it with DNase I (Invitrogen). RNA from cultured cells was isolated using RNA-extraction kit (Sigma, St Louis, MO). Random primers (Invitrogen) and super-script II (Invitrogen) were used to synthesize first-strand complementary DNAs from equivalent amounts of RNA from each sample. Real-time RT-PCR was performed in a 7000 Real-Time PCR System (Applied Biosystems, Grand Island, NY) with SYBR Green PCR Master Mix (Applied Biosystems). Data were generated by the comparative threshold cycle $(\Delta \mathrm{CT})$ method by normalizing to hypoxanthine-guanine phosphoribosyltransferase. The sequences of the primers are available upon request.

Plaque assay measuring RSV virus. Supernatant of BALF was serially diluted with serum-free MEM (Cellgro, Manassas, VA) on ice, and then were inoculated onto $90 \%$ confluent HEp-2 cells in six-well plate. After $90 \mathrm{~min}$ of incubation at $37^{\circ} \mathrm{C}$, the inoculant was removed and supplemented with 1:1 mix of complete $2 \times$ EMEM (Lonza, Walkersville, $\mathrm{MD}$ ) and $1 \%$ low meting point agarose (Promega, Madison, MA). Four days later, the plate was fixed with $1 \%$ formalin-phosphate-buffered saline, and stained with $0.05 \%$ neutral red (Fisher). The plaques were counted and viral load was expressed as the number of p.f.u. $\mathrm{ml}^{-1}$ of BALF.
Tfh, germinal center B cells and RSV-specific IgG measurement. Mediastinal lymph nodes cell suspensions of RSV-infected mice were used for detection of Tfh and germinal center B cells. Tfh cells were defined as $\mathrm{CD}^{+}{ }^{+} \mathrm{PD}-1^{+} \mathrm{CXCR}^{+}$cells, and germinal center $\mathrm{B}$ cells were defined as $\mathrm{B} 220^{+} \mathrm{CD} 95^{+} \mathrm{GL}^{+} 7^{+}$cells. For the detection of RSV-specific IgG, we coated ELISA plates (Themo Scientific, Rochester, NY) with diluted virus overnight at $4{ }^{\circ} \mathrm{C}$. Following blockade of non-specific binding, serially diluted serum and BALF was then incubated with coated RSV for $2 \mathrm{~h}$ at room temperature, and then detected with secondary biotin-conjugated anti-mouse IgG (eBioscience, San Diego, CA) followed by Avidin-horseradish peroxidase (Biolegend) and TMB substrate solution (Biolegend). The absorbance was read at $450 \mathrm{~nm}$ by spectrophotometer.

Intracellular cytokine staining. Measurement of IL-10 and IFN- $\gamma$ producing cells in vivo was based on a previously described protocol. ${ }^{16,53}$ Briefly, at indicated days post RSV infection, mice were injected IV with $500 \mu \mathrm{l}$ of a phosphate-buffered saline solution containing $750 \mu \mathrm{g}$ Monensin (Sigma) $6 \mathrm{~h}$ before harvesting. Lung single cell suspensions were prepared in the presence of monensin. Cells were then fixed and permeablized and intracellular IL-10 and IFN- $\gamma$ staining as described. ${ }^{16,53}$ Lung single cell suspensions were generated as described. ${ }^{53}$ Lung cells were subsequently re-stimulated with PMA $\left(100 \mathrm{ng} \mathrm{ml}^{-1}\right)$ and ionomycin $\left(1 \mu \mathrm{g} \mathrm{ml}^{-1}\right)$ in the presence of Golgi-Stop $\left(1 \mu \mathrm{ml}^{-1}\right)$ for $4 \mathrm{~h}$. Then cells were fixed and permeablized using the Cyto-Fix and Perm-Wash system (BD Biosciences). Cell surface CD8 ${ }^{+}$, and intracellular IL-10 and IFN- $\gamma$ were stained accordingly. ${ }^{16,53}$

Generation of mixed BM chimeras. WT mice were lethally irradiated $(1,100 \mathrm{rad})$ and IV injected with the 1:1 mixed BM cells from WT $\left(\mathrm{CD} 45.1^{+}\right)$mice and congenic BM cells from either IRF1- or STAT1deficient $\left(\mathrm{CD} 45.2^{+}\right)$mice. After 12 weeks for reconstitution, the chimeric mice were then infected with RSV A2 virus.

Construction of IRF1-expressing retrovirus. The full-length mouse IRF1 was amplified by PCR from a plasmid containing the complementary DNA of IRF1 (ATCC) using the primers: IRF1-F1 ( $5^{\prime}-\mathrm{GC}$ CACCATGCCAATCACTCGAATG- $\left.3^{\prime}\right)$ and IRF1-R1 (5'-GTTGTTG TCGACCTATGGTGCACAAGGAATGGCCT-3'). Primer IRF1-F1 contains no enzyme restriction site, whereas primer IRF1-R1 contains a restriction site for SalI. The PCR product was then digested with SalI, gel purified, and ligated into the pre-linearized retroviral vector backbone encoding a marker of Thy1.1 (MSCV-Thy1.1), which contains a blunt end at $5^{\prime}$ and a SalI site at $3^{\prime}$. This ligation thus generated a bicistronic retrovirus encoding both IRF1 and Thy1.1 (MSCV-IRF1Thy1.1). The fidelity of MSCV-IRF1-Thy1.1 was confirmed to be identical to the sequence deposited in the gene bank.

Retroviral transduction of BMDCs. BMDCs were generated as described and cultured in presence of granulocyte-macrophage colony-stimulating factor $\left(20 \mathrm{ng} \mathrm{ml}^{-1}\right) \cdot{ }^{54}$ At days 2 and 3 of culture, cells were transduced with bicistronic retrovirus expressing Thy1.1 only (MSCV-Thy1.1, control), or MSCV-IRF1-Thy1.1 (IRF1) through spin infection (2,500 r.p.m.; 2h) in presence of polybrene $\left(10 \mu \mathrm{g} \mathrm{ml}^{-1}\right), 2-\mathrm{ME}$, and Hepes. ${ }^{55}$ Viral supernatant was replaced with the former culture after transduction. On day 5, transduced BMDCs $\left(\right.$ Thy $1.1^{+}$) were analyzed by flow cytometry for PD-L1 expression.

Flow cytometry analysis. All fluorescence-activated cell sorting (FACS) antibodies were purchased from Biolegend, BD Biosciences or eBioscience. Cells were acquired through FACSCalibur, FACSCanto, or LSR II apparatus (BD Biosciences). Data were then analyzed by using FlowJo software (Treestar, Ashland, OR).

Statistical analyses. Data are reported as mean \pm s.e.m. Analysis of variance, parametric (paired or unpaired Student's $t$-test) or nonparametric (Mann-Whitney test) $t$-tests were used, where noted. $P$-values $>0.05$ are considered not significant. 
SUPPLEMENTARY MATERIAL is linked to the online version of the paper at http://www.nature.com/mi

\section{ACKNOWLEDGMENTS}

We thank the parents and subjects who participated in the study. We thank Drs Mark Kaplan, Matthew Hufford, and Matthew Olson for critical reading of the manuscript. We thank Drs W. Zhang, X. Fu, and L. Chen for critical reagents. This work was supported by Showalter Trust Funds to J.S. and American Lung Association Postdoctoral Fellowship Award (RT-310817) to S.Y.

\section{DISCLOSURE}

The authors declared not conflict of interest.

c 2015 Society for Mucosal Immunology

\section{REFERENCES}

1. Smyth, R.L. \& Openshaw, P.J. Bronchiolitis. Lancet 368, 312-322 (2006).

2. Falsey, A.R., Hennessey, P.A., Formica, M.A., Cox, C. \& Walsh, E.E. Respiratory syncytial virus infection in elderly and high-risk adults. N. Engl. J. Med. 352, 1749-1759 (2005).

3. Graham, B.S., Johnson, T.R. \& Peebles, R.S. Immune-mediated disease pathogenesis in respiratory syncytial virus infection. Immunopharmacology 48, 237-247 (2000).

4. Peebles, R.S. Jr \& Graham, B.S. Pathogenesis of respiratory syncytial virus infection in the murine model. Proc. Am. Thorac. Soc. 2, 110-115 (2005).

5. Bennett, B.L. et al. Immunopathogenesis of respiratory syncytial virus bronchiolitis. J. Infect. Dis. 195, 1532-1540 (2007).

6. Miyairi, I. \& DeVincenzo, J.P. Human genetic factors and respiratory syncytial virus disease severity. Clin. Microbiol. Rev. 21, 686-703 (2008).

7. Oshansky, C.M., Zhang, W., Moore, E. \& Tripp, R.A. The host response and molecular pathogenesis associated with respiratory syncytial virus infection. Future Microbiol. 4, 279-297 (2009).

8. Malley, R. et al. Reduction of respiratory syncytial virus (RSV) in tracheal aspirates in intubated infants by use of humanized monoclonal antibody to RSV F protein. J. Infect. Dis. 178, 1555-1561 (1998).

9. Collins, P.L. \& Graham, B.S. Viral and host factors in human respiratory syncytial virus pathogenesis. J. Virol. 82, 2040-2055 (2008).

10. Ruckwardt, T.J., Bonaparte, K.L., Nason, M.C. \& Graham, B.S. Regulatory $T$ cells promote early influx of CD8 + T cells in the lungs of respiratory syncytial virus-infected mice and diminish immunodominance disparities. J. Virol. 83, 3019-3028 (2009).

11. Fulton, R.B., Meyerholz, D.K.\& Varga, S.M. Foxp3 + CD4 regulatory Tcells limit pulmonary immunopathology by modulating the CD8 T cell response during respiratory syncytial virus infection. J. Immunol. 185, 2382-2392 (2010).

12. Lee, D.C. etal. CD25 + natural regulatory Tcells are critical in limiting innate and adaptive immunity and resolving disease following respiratory syncytial virus infection. J. Virol. 84, 8790-8798 (2010).

13. Loebbermann, J. et al. Regulatory T cells expressing granzyme B play a critical role in controlling lung inflammation during acute viral infection. Mucosal Immunol. 5, 161-172 (2012).

14. Loebbermann, J. et al. IL-10 regulates viral lung immunopathology during acute respiratory syncytial virus infection in mice. PLoS One 7, e32371 (2012).

15. Weiss, K.A., Christiaansen, A.F., Fulton, R.B., Meyerholz, D.K. \& Varga, S.M. Multiple CD4 + T cell subsets produce immunomodulatory IL-10 during respiratory syncytial virus infection. J. Immunol. 187, 3145-3154 (2011).

16. Sun, J. et al. Autocrine regulation of pulmonary inflammation by effector T-cell derived IL-10 during infection with respiratory syncytial virus. PLoS Pathog. 7, e1002173 (2011).

17. Gentile, D.A. et al. Cytokine gene polymorphisms moderate illness severity in infants with respiratory syncytial virus infection. Hum. Immunol. 64, 338-344 (2003).

18. Hoebee, B. et al. Influence of promoter variants of interleukin-10, interleukin-9, and tumor necrosis factor-alpha genes on respiratory syncytial virus bronchiolitis. J. Infect. Dis. 189, 239-247 (2004).
19. Francisco, L.M., Sage, P.T. \& Sharpe, A.H. The PD-1 pathway in tolerance and autoimmunity. Immunol. Rev. 236, 219-242 (2010).

20. Barber, D.L. et al. Restoring function in exhausted CD8 T cells during chronic viral infection. Nature 439, 682-687 (2006).

21. Virgin, H.W., Wherry, E.J. \& Ahmed, R. Redefining chronic viral infection. Cell 138, 30-50 (2009).

22. Wherry, E.J. T cell exhaustion. Nat. Immunol. 12, 492-499 (2011).

23. Brown, K.E., Freeman, G.J., Wherry, E.J. \& Sharpe, A.H. Role of PD-1 in regulating acute infections. Curr. Opin. Immunol. 22, 397-401 (2010).

24. Erickson, J.J. et al. Viral acute lower respiratory infections impair CD8 + T cells through PD-1. J. Clin. Invest. 122, 2967-2982 (2012).

25. McNally, B., Ye, F., Willette, M. \& Flano, E. Local blockade of epithelial PDL1 in the airways enhances $T$ cell function and viral clearance during influenza virus infection. J. Virol. 87, 12916-12924 (2013).

26. Rutigliano, J.A. et al. Highly pathological influenza A virus infection is associated with augmented expression of PD-1 by functionally compromised virus-specific CD8 + T cells. J. Virol. 88, 1636-1651 (2014).

27. Telcian, A.G. et al. RSV-induced bronchial epithelial cell PD-L1 expression inhibits CD8 + Tcell nonspecific antiviral activity. J. Infect. Dis. 203, 85-94 (2011).

28. Goldberg, M.V. et al. Role of PD-1 and its ligand, $\mathrm{B} 7-\mathrm{H} 1$, in early fate decisions of CD8 T cells. Blood 110, 186-192 (2007).

29. Crowe, C.R. et al. Critical role of IL-17RA in immunopathology of influenza infection. J. Immunol. 183, 5301-5310 (2009).

30. Polack, F.P. et al. A role for immune complexes in enhanced respiratory syncytial virus disease. J. Exp. Med. 196, 859-865 (2002).

31. Delgado, M.F. et al. Lack of antibody affinity maturation due to poor Toll-like receptor stimulation leads to enhanced respiratory syncytial virus disease. Nat. Med. 15, 34-41 (2009).

32. Crotty, S. Follicular helper CD4 T cells (TFH). Annu. Rev. Immunol. 29, 621-663 (2011).

33. Lambrecht, B.N. \& Hammad, H. Lung dendritic cells in respiratory viral infection and asthma: from protection to immunopathology. Annu. Rev. Immunol. 30, 243-270 (2012).

34. Hespel, C. \& Moser, M. Role of inflammatory dendritic cells in innate and adaptive immunity. Eur. J. Immunol. 42, 2535-2543 (2012).

35. Hufford, M.M., Kim, T.S., Sun, J. \& Braciale, T.J. Antiviral CD8 + T cell effector activities in situ are regulated by target cell type. J. Exp. Med. 208, 167-180 (2011).

36. Yamazaki, T. et al. Expression of programmed death 1 ligands by murine $T$ cells and APC. J. Immunol. 169, 5538-5545 (2002).

37. Schreiner, B. et al. Interferon-beta enhances monocyte and dendritic cell expression of B7-H1 (PD-L1), a strong inhibitor of autologous T-cell activation: relevance for the immune modulatory effect in multiple sclerosis. J. Neuroimmunol. 155, 172-182 (2004).

38. Au-Yeung, N., Mandhana, R. \& Horvath, C.M. Transcriptional regulation by STAT1 and STAT2 in the interferon JAK-STAT pathway. JAKSTAT 2, e23931 (2013).

39. Lee, S.J. et al. Interferon regulatory factor-1 is prerequisite to the constitutive expression and IFN-gamma-induced upregulation of B7H1 (CD274). FEBS lett. 580, 755-762 (2006).

40. Matsuyama, T. et al. Targeted disruption of IRF-1 or IRF-2 results in abnormal type I IFN gene induction and aberrant lymphocyte development. Cell 75, 83-97 (1993).

41. Shin, H. et al. A role for the transcriptional repressor Blimp-1 in CD8(+) $\mathrm{T}$ cell exhaustion during chronic viral infection. Immunity 31, 309-320 (2009).

42. Utzschneider, D.T. et al. T cells maintain an exhausted phenotype after antigen withdrawal and population reexpansion. Nat. Immunol. 14, 603-610 (2013).

43. Wilson, J. et al. Genetic variation at the IL10 gene locus is associated with severity of respiratory syncytial virus bronchiolitis. J. Infect. Dis. 191, 1705-1709 (2005)

44. Singh, A.K., Stock, P. \& Akbari, O. Role of PD-L1 and PD-L2 in allergic diseases and asthma. Allergy 66, 155-162 (2011).

45. Lukacs, N.W. et al. Differential immune responses and pulmonary pathophysiology are induced by two different strains of respiratory syncytial virus. Am. J. Pathol. 169, 977-986 (2006). 
46. Stokes, K.L. et al. Differential pathogenesis of respiratory syncytial virus clinical isolates in BALB/c mice. J. Virol. 85, 5782-5793 (2011).

47. Ostler, T., Davidson, W. \& Ehl, S. Virus clearance and immunopathology by CD8 $(+)$ Tcells during infection with respiratory syncytial virus are mediated by IFN-gamma. Eur. J. Immunol. 32, 2117-2123 (2002).

48. Rutigliano, J.A. \& Graham, B.S. Prolonged production of TNF-alpha exacerbates illness during respiratory syncytial virus infection. J. Immunol. 173, 3408-3417 (2004).

49. Braciale, T.J., Sun, J. \& Kim, T.S. Regulating the adaptive immune response to respiratory virus infection. Nat. Rev. Immunol. 12, 295-305 (2012).

50. Valitutti, S., Muller, S., Dessing, M. \& Lanzavecchia, A. Different responses are elicited in cytotoxic $\mathrm{T}$ lymphocytes by different levels of T cell receptor occupancy. J. Exp. Med. 183, 1917-1921 (1996).
51. Moore, M.L. et al. A chimeric A2 strain of respiratory syncytial virus (RSV) with the fusion protein of RSV strain line 19 exhibits enhanced viral load, mucus, and airway dysfunction. J. Virol. 83, 4185-4194 (2009).

52. Sun, J., Dodd, H., Moser, E.K., Sharma, R. \& Braciale, T.J. CD4 + T cell help and innate-derived IL-27 induce Blimp-1-dependent IL-10 production by antiviral CTLs. Nat. Immunol. 12, 327-334 (2011).

53. Sun, J., Madan, R., Karp, C.L. \& Braciale, T.J. Effector T cells control lung inflammation during acute influenza virus infection by producing IL-10. Nat. Med. 15, 277-284 (2009).

54. Sun, J. \& Pearce, E.J. Suppression of early IL-4 production underlies the failure of CD4 T cells activated by TLR-stimulated dendritic cells to differentiate into Th2 cells. J. Immunol. 178, 1635-1644 (2007).

55. Sun, J., Krawczyk, C.J. \& Pearce, E.J. Suppression of Th2 cell development by Notch ligands Delta1 and Delta4. J. Immunol. 180, 1655-1661 (2008). 